\title{
Propriedades mecânicas de materiais compósitos à base de cimento Portland e resina epoxi
}

\section{(Mechanical properties of composite materials based on Portland cement and epoxy resin)}

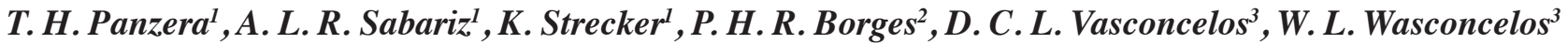 \\ ${ }^{I}$ Departamento de Engenharia Mecânica, Universidade Federal de S. João del-Rei - UFSJ, Campus S. Antônio \\ Praça Frei Orlando 170, S. J. del-Rei, MG, Brasil 36307-352 \\ ${ }^{2}$ Monier Technical Centre Ltd., Sussex Manor Business Park, Gatwick Road, Crawley, RH10 9NZ, Inglaterra \\ ${ }^{3}$ Departamento de Engenharia Metalúrgica e de Materiais, Universidade Federal de Minas Gerais, Escola de \\ Engenharia, Rua Espírito Santo 35, sala 206, Centro, Belo Horizonte, MG, Brasil 30160-030 \\ panzera@ufsj.edu.br
}

\begin{abstract}
Resumo
O estudo de materiais de alto desempenho e multifuncionais, como os compósitos poliméricos cimentícios, tem sido o foco de inúmeras pesquisas na indústria da construção civil. Este trabalho investiga o efeito da combinação de uma fase polimérica termorrígida, uma resina epóxi, com cimento Portland branco estrutural, seguido da avaliação da resistência à compressão e módulo de elasticidade. Este compósito, quando comparado individualmente com as suas matérias-prima originais, promove um aumento da resistência mecânica à compressão, redução da massa específica e, também uma mudança significativa do comportamento mecânico. As mudanças nas propriedades mecânicas estão associadas à hidratação da fase cimentícia na presença da resina, fato comprovado através da análise espectroscópica na região do infravermelho.

Palavras-chave: compósito cimentício polimérico, resina epóxi, cimento Portland branco, espectroscopia por infravermelho.
\end{abstract}

Abstract

The study of multi-functional materials of high performance, as the polymeric-cementitious composites, has been the focus of several researches in the industry of the civil engineering. This work investigates the effect of the combination of a thermorigid epoxy phase and the white Portland cement, followed by the evaluation of its compressive strength and modulus of elasticity. This composite, when the phases are individually compared, provides an increase of the compressive strength, a reduction of the density, and a significant change of the mechanical behaviour. The changes in mechanical behaviour are associated with the hydration of cement in the presence of resin, which was evident after infrared spectroscopy analysis.

Keywords: polymeric cementitious composite, epoxy resin, white Portland cement, infrared spectroscopy (FTIR).

\section{INTRODUÇÃO}

A utilização e o desenvolvimento de produtos cimentícios poliméricos vêm sendo conduzidos em vários países há mais de quarenta anos. A adição polimérica à pasta cimentícia tornou-se o foco de inúmeras pesquisas no Japão e Europa na década de 70 e, posteriormente, na década de 80 nos Estados Unidos [1-2]. Estes compósitos vêm sendo empregados na construção civil tanto na fase de acabamento, como também na fabricação de produtos pré-fabricados. Recentemente, uma nova demanda por compósitos poliméricos ressurgiu, e um exemplo claro disso é a crescente produção de mármores e granitos artificiais a cada ano. Esta atividade vem sendo impulsionada pelos conceitos de sustentabilidade do século XXI, uma vez que o uso de rochas minerais para confecção de revestimentos na construção civil promove não só o consumo da fonte mineral, mas também traz sérios problemas ambientais [3-
4]. Em termos de microestrutura, a fase polimérica tende a recobrir os grãos não hidratados de cimento, retardando o processo de hidratação parcialmente ou completamente. Em alguns casos, promove maior formação de produtos internos do que produtos externos de $\mathrm{C}_{3} \mathrm{~S}$, além de evitar a formação de cristais de etringita durante o processo inicial de hidratação [5-7]. Porém, é bem possível que a maior vantagem desses sistemas seja a redução da porosidade, com conseqüente diminuição dos caminhos livres para propagação de fissuras e aumento da resistência mecânica final [8].

Diversos tipos de compósitos poliméricos cimentícios foram revisados [1,2]. De um modo geral, um percentual de água é adicionado aos compósitos com o intuito de promover a hidratação dos grãos cimentícios. O presente trabalho difere dos demais, uma vez que investiga o efeito da adição de um polímero termorrígido de alta resistência mecânica à fase cimentícia sem adição de água. Somente uma formulação de 
referência, isto é, pasta de cimento puro, foi confeccionada com água para efeito de comparação. As propriedades mecânicas investigadas foram resistência à compressão e módulo de elasticidade. Também foram avaliadas as massas específicas correspondentes. O comportamento mecânico da curva tensão-deformação dos compósitos no ensaio de compressão será apresentado e discutido. Finalmente, a análise por espectrometria na região do infravermelho foi utilizada para confirmação de reações químicas entre a fase cimentícia e a resina.

\section{MATERIAIS E MÉTODOS}

As condições experimentais analisadas nesta investigação podem ser observadas na Tabela I.

Tabela I - Condições experimentais. [Table I - Experimental conditions.]

\begin{tabular}{cccc}
\hline & $\begin{array}{c}\text { Polímero } \\
(\%)\end{array}$ & $\begin{array}{c}\text { Cimento } \\
(\%)\end{array}$ & $\begin{array}{c}\text { Água } \\
(\%)\end{array}$ \\
\hline C1 & 100 & 0 & 0 \\
C2 & 75 & 25 & 0 \\
C3 & 50 & 50 & 0 \\
C4 & 25 & 75 & 0 \\
C5 & 0 & 100 & 30 \\
\hline
\end{tabular}

Cinco tipos de compósitos foram preparados, representando as variações de fração das fases cimentícia/ polimérica adotadas neste estudo. $\mathrm{O}$ compósito $\mathrm{C} 1$ foi preparado com $100 \%$ de fase polimérica e o compósito C5 com $100 \%$ de fase cimentícia. Os demais compósitos C2, C3 e C4 foram fabricados com $75 \%, 50 \%$ e $25 \%$ de fase polimérica, respectivamente. Cabe ressaltar que a adição de água foi realizada apenas no compósito $\mathrm{C} 5$, constituído apenas de cimento. O fator água-cimento foi de 30\% sobre a massa de cimento Portland. A Fig. 1 exibe uma foto dos compósitos investigados (C1 a C5).

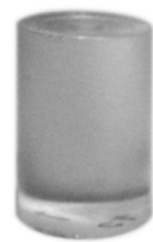

C1

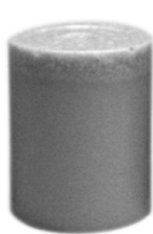

C2

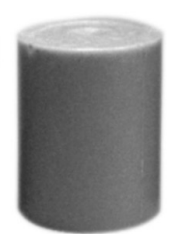

C3

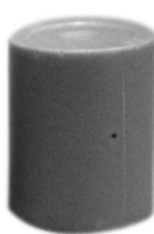

C4

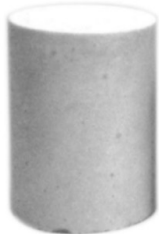

C5
Figura 1: Foto dos compósitos investigados no experimento. [Figure 1: Picture of the studied composite materials.]

\section{Fase cimentícia: cimento Portland}

O cimento Portland utilizado no experimento foi o CPB40 (NBR 12989) [9] do fabricante Cauê, indústria brasileira. A Tabela II exibe a análise química deste material.
Tabela II - Análise química do cimento CPB-40. [Table II - Chemical analysis of the CPB-40 cement.]

\begin{tabular}{cc}
\hline \multicolumn{2}{c}{ Cimento Portland Branco - Cauê } \\
\hline $\mathrm{SiO}_{2}$ & 23,59 \\
$\mathrm{Al}_{2} \mathrm{O}_{3}$ & 7,52 \\
$\mathrm{Fe}_{2} \mathrm{O}_{3}$ & 2,48 \\
$\mathrm{CaO}$ & 55,85 \\
$\mathrm{MgO}$ & 2,96 \\
$\mathrm{SO}_{3}$ & 1,47 \\
$\mathrm{Na}_{2} \mathrm{O}$ & 0,37 \\
$\mathrm{~K}_{2} \mathrm{O}$ & 0,89 \\
$\mathrm{CO}_{2}$ & 4,87 \\
Perda ao Fogo $(\%)$ & 5,08 \\
Blaine $\left(\mathrm{cm}^{2} / \mathrm{g}\right)$ & 5004 \\
\hline
\end{tabular}

(Fonte: Cimentos Cauê)

Fase polimérica termorrígida: resina epóxi

A fase polimérica termorrígida utilizada neste trabalho foi uma resina epóxi de alto desempenho, fabricada pela Indústria Química Huntsman. A resina epóxi é constituída de duas partes, sendo uma denominada araldite e a outra endurecedor. O araldite usado foi o LY 1564BR e o endurecedor o Aradur 2954. A proporção da mistura em massa utilizada foi de $74 \%$ de araldite para $26 \%$ de endurecedor. A viscosidade, a massa específica e o tempo de cura total foram fornecidos pelo fabricante, (ver Tabela III).

Tabela III - Característica da resina epoxi.

[Table III - Characteristics of the epoxy resin.]

\begin{tabular}{ccc}
\hline & LY 1564BR & Aradur 2954 \\
\hline $\begin{array}{c}\text { Viscosidade a } \\
25{ }^{\circ} \mathrm{C}(\mathrm{mPas})\end{array}$ & $1200-1400$ & $70-120$ \\
$\begin{array}{c}\text { Massa específica } \\
\left(\mathrm{g} / \mathrm{cm}^{3}\right)\end{array}$ & $1,10-1,20$ & $0,94-0,95$ \\
$\begin{array}{c}\text { Proporção em massa } \\
(\%)\end{array}$ & 74 & 26 \\
$\begin{array}{c}\text { Tempo de cura da } \\
\text { mistura }\end{array}$ & \multicolumn{2}{c}{7 dias } \\
\hline
\end{tabular}

(Fonte: Hunstman)

\section{Preparação dos corpos de prova}

Sete corpos de prova de cada condição experimental foram fabricados utilizando moldes cilíndricos poliméricos de dimensão 29,88 mm de diâmetro e 42,00 $\mathrm{mm}$ de altura e uma bancada para vibração e compactação dos mesmos. Após o período de cura de 28 dias, foram desmoldados e cortados, garantindo o paralelismo necessário para execução do ensaio de resistência à compressão, e para determinação das massas específicas dos mesmos. A Fig. 2 mostra o 
processo de corte dos corpos de prova.

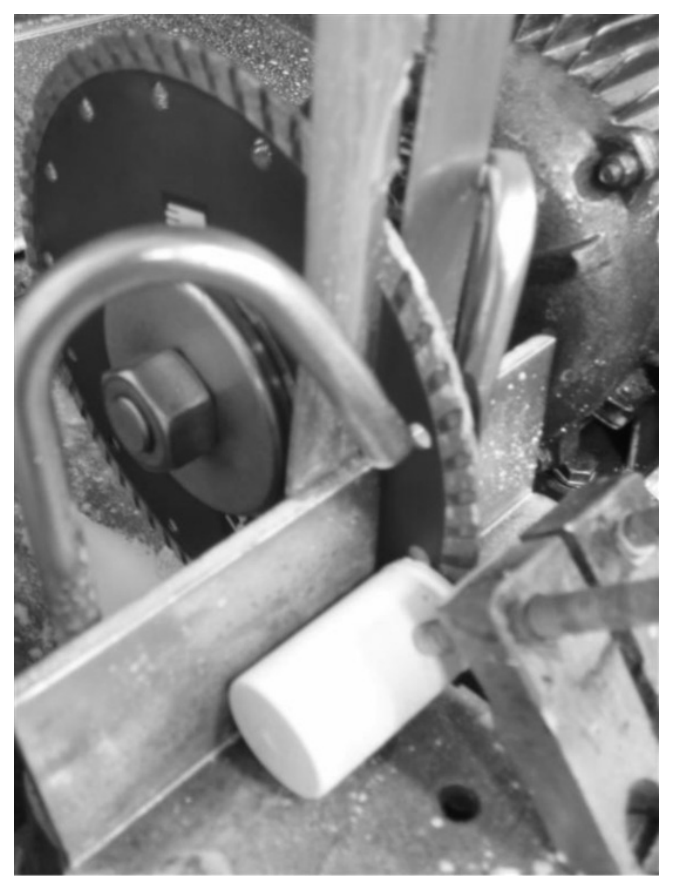

Figura 2: Corte dos corpos de prova dos compósitos estudados. [Figure 2: Cutting the composite specimens.]

\section{Massa específica e propriedades mecânicas}

O ensaio de resistência mecânica à compressão foi baseado nas recomendações da norma britânica BS-12390 [10]. Os testes foram realizados de forma aleatória. Os compósitos investigados foram rompidos para um período de cura de 28 dias. Os módulos de elasticidade foram determinados em função dos gráficos tensão-deformação obtidos no ensaio de compressão. A massa específica dos compósitos foi calculada dividindo a massa do compósito seco (após $24 \mathrm{~h}$ na estufa e a $105^{\circ} \mathrm{C}$ ) pelo volume das amostras.

\section{Espectroscopia de infravermelho (FT-IR)}

Os compósitos $\mathrm{C} 1$ a $\mathrm{C} 5$ foram analisados por espectroscopia na região do infravermelho por transformada de Fourier (FTIR) e a técnica utilizada foi a de transmissão com o uso de pastilhas de $\mathrm{KBr}$. Cada amostra foi inicialmente pulverizada em um gral de ágata, dispersas em $\mathrm{KBr}$ e em seguida prensadas e analisadas em um equipamento Perkin-Elmer Spectrum 1000. Os espectros foram traçados no intervalo de comprimento de onda entre 4000 a 2400 $\mathrm{cm}^{-1}$ e a intensidade expressa em transmitância (\%T). Os principais produtos hidratação do cimento Portland são os silicatos de cálcio hidratados (C-S-H) e hidróxido de cálcio ou portlandita $(\mathrm{CH})$. Portanto, as bandas referentes à água constituinte e absorvida pelos silicatos e sulfoaluminatos de cálcio (banda larga centrada em $3460 \mathrm{~cm}^{-1}$ ) e referente à hidroxila $\left(\mathrm{OH}^{-}\right)$constituinte da portlandita $\left(3640 \mathrm{~cm}^{-1}\right)$ podem ser utilizadas para verificar se o cimento Portland branco hidratou na presença da resina epoxídica nos compósitos C2, C3 e C4.

\section{RESULTADOS E DISCUSSÃO}

\section{Massa específica e propriedades mecânicas}

A Tabela IV exibe as médias e os desvios padrão DP dos resultados de resistência à compressão Rc, módulo de elasticidade E e massa específica $\rho$ para as condições experimentais investigadas.

Os resultados experimentais foram agrupados em pares a fim de permitir uma comparação entre as propriedades e as condições experimentais, sendo apresentados nos gráficos das Figs. 3 a 6. A Fig. 3 exibe o gráfico das propriedades resistência à compressão $(\mathrm{MPa})$ e massa específica $\left(\mathrm{g} / \mathrm{cm}^{3}\right)$. É possível observar pela variação da massa específica que os compósitos fabricados com $100 \%$ de polímero (C1) e $100 \%$ de cimento (C5) exibem, respectivamente, os limites inferiores $\left(1,17 \mathrm{~g} / \mathrm{cm}^{3}\right)$ e superiores $\left(1,92 \mathrm{~g} / \mathrm{cm}^{3}\right)$ obtidos, representando uma variação percentual de $64,10 \%$. Os outros compósitos apresentam valores de massas específicas condizentes com a regra da mistura das fases presentes. Por outro lado, os resultados de resistência à compressão não exibem o mesmo comportamento. Apesar do resultado de resistência à compressão do compósito polimérico C1 $(64,33 \mathrm{MPa})$ ser superior ao compósito cimentício C5 $(28,93 \mathrm{MPa})$, a mesma variação não é observada para os demais compósitos, visto que a maior resistência mecânica é apresentada pelo compósito C3 (98,80 MPa), constituído de $50 \%$ de fase polimérica e $50 \%$ de fase cimentícia. A relação resistência mecânica e massa específica apresentada

Tabela IV - Resultados experimentais médios e desvios-padrão.

[Table V - Average experimental results and standard deviations (DP).]

\begin{tabular}{|c|c|c|c|c|c|c|}
\hline & $\mathrm{Rc}[\mathrm{MPa}]$ & $\mathrm{DP}[\mathrm{MPa}]$ & $\mathrm{E}$ [GPa] & $\mathrm{DP}[\mathrm{GPa}]$ & $\rho\left[\mathrm{g} / \mathrm{cm}^{3}\right]$ & $\mathrm{DP}\left[\mathrm{g} / \mathrm{cm}^{3}\right]$ \\
\hline $\mathrm{C} 1$ & 64,33 & 0,23 & 23,76 & 1,43 & 1,17 & 0,01 \\
\hline $\mathrm{C} 2$ & 67,07 & 0,56 & 25,29 & 1,52 & 1,34 & 0,02 \\
\hline $\mathrm{C} 3$ & 98,80 & 2,11 & 33,98 & 4,69 & 1,64 & 0,01 \\
\hline $\mathrm{C} 4$ & 81,73 & 2,55 & 46,27 & 4,65 & 1,84 & 0,02 \\
\hline C5 & 28,93 & 1,90 & 47,88 & 1,63 & 1,92 & 0,03 \\
\hline
\end{tabular}




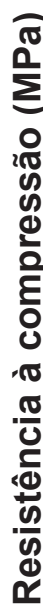

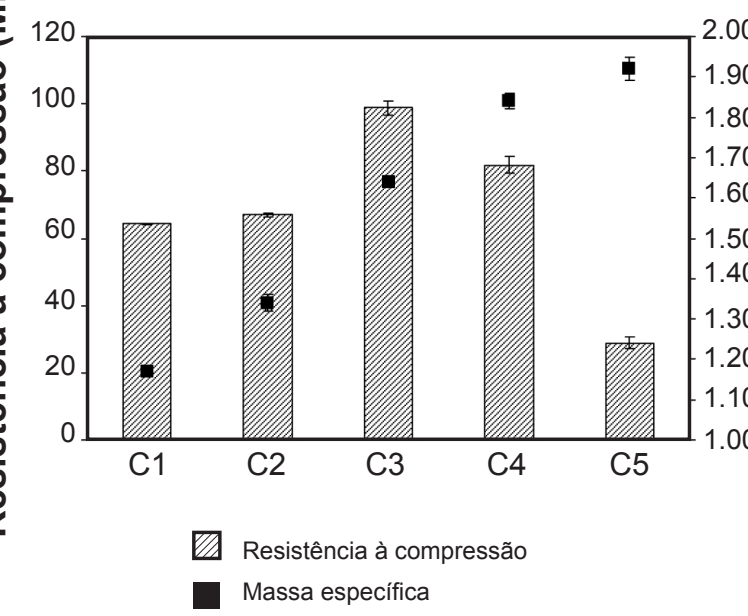

Figura 3: Resistência à compressão e massa específica.

[Figure 3: Compressive strength and specific mass.]

pelo compósito C3 é bastante promissora, exibindo elevada resistência mecânica e massa específica relativamente baixa, comparado com os compósitos C4 e C5.

A Fig. 4 mostra a relação entre as propriedades módulo de elasticidade $(\mathrm{GPa})$ e resistência à compressão $(\mathrm{MPa})$. A fase polimérica $\mathrm{C} 1 \quad(23,76 \mathrm{GPa})$ exibe módulo de elasticidade inferior aos resultados apresentado pela fase cimentícia C5 (47,88 GPa), com uma diferença percentual de aproximadamente $101,51 \%$. Observando estas propriedades para os compósitos estudados, verifica-se que a fase cimentícia proporciona o aumento do módulo de elasticidade do compósito, enquanto a fase polimérica contribui para o aumento da resistência à compressão. Conforme discutido anteriormente, o valor ótimo de resistência mecânica é apresentado pelo compósito C3 (mistura 50/50).

A Fig. 5 apresenta a correlação entre as propriedades massa específica $\rho$ e módulo de elasticidade $\mathrm{E}$ dos

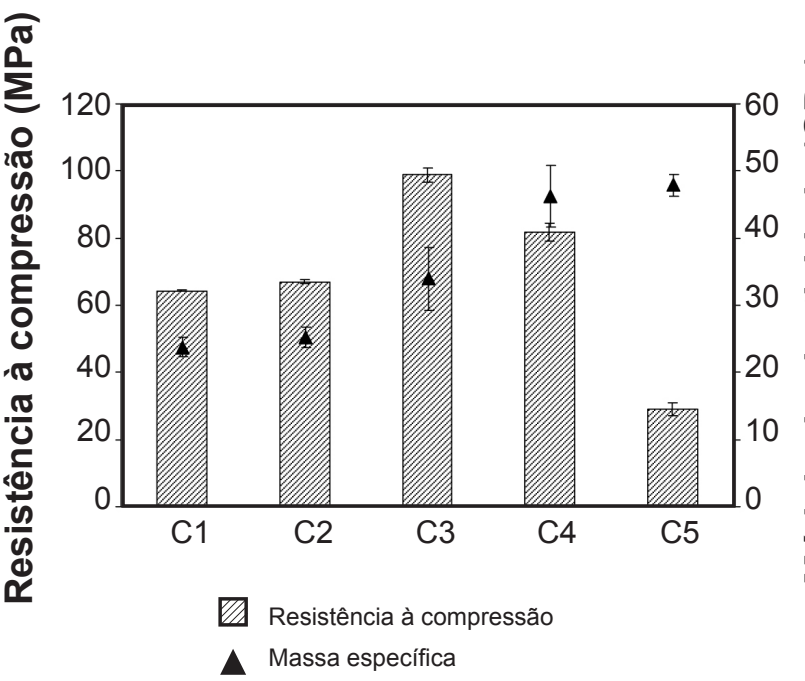

Figura 4: Módulo de elasticidade e resistência à compressão. [Figure 4: Elasticity moduluis and compressive strength.]

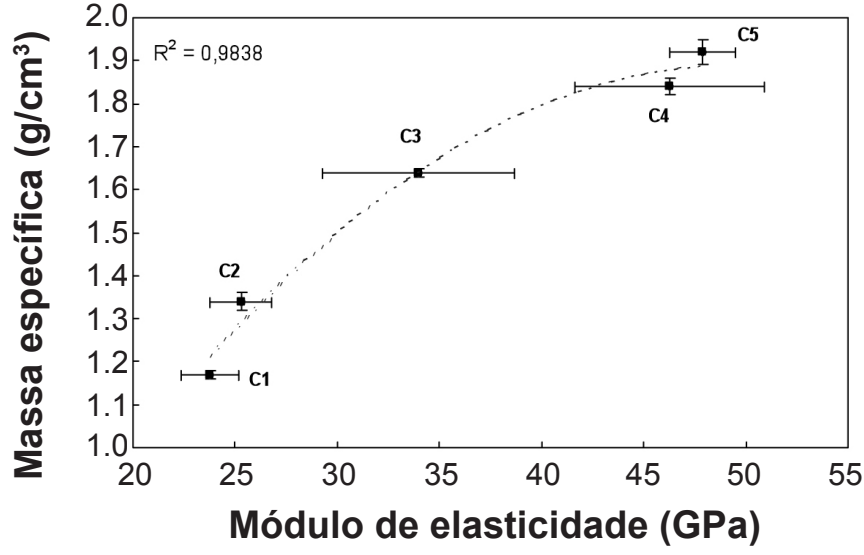

Figura 5: Correlação entre massa específica e módulo de elasticidade.

[Figure 5: Specific mass as a function of the elasticity modulus.]

compósitos. Existe uma correlação polinomial da ordem de segundo grau entre estas variáveis, representada pela equação $\rho=-0,0021 \mathrm{E}^{2}+0,21 \mathrm{E}-1,3656$ com coeficiente de correlação de $\mathrm{R}^{2}=0,9838$. Verifica-se o aumento do módulo de elasticidade em função do aumento da massa específica dos compósitos investigados.

Observou-se nos gráficos anteriores (Figs. 3 a 5) um comportamento distinto das fases poliméricas e cimentícias. Constatou-se que a massa específica apresenta uma correlação direta com o módulo de elasticidade, por outro lado a resistência mecânica não segue a mesma tendência. Isto pode ser melhor compreendido plotando o gráfico tensãodeformação obtido no ensaio de resistência à compressão, a fim de verificar o comportamento mecânico apresentado pelos compósitos $\mathrm{C} 1$ a C5 (Fig. 6).

Nota-se uma variação da inclinação da curva (módulo de elasticidade) em função da fase presente, assim como observado na Fig. 4. A presença da fase cimentícia promove um aumento do módulo de elasticidade, exibindo um comportamento de material cerâmico evidenciado, principalmente, pela fratura frágil do compósito C5. O

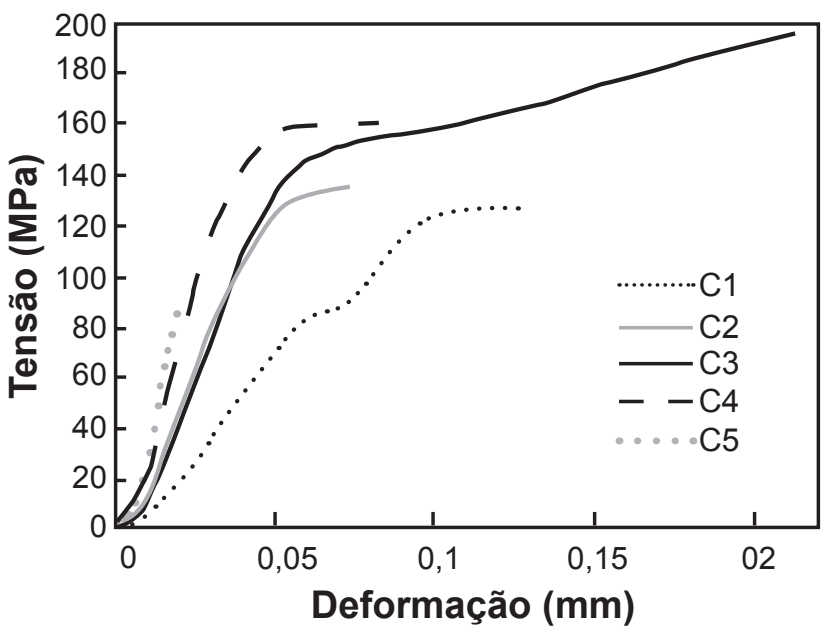

Figura 6: Curvas tensão-deformação dos compósitos. [Figure 6: Stress-strain curves of the composites.] 
compósito C3 constituído de 50\% de ambas as fases cimentícia-polimérica destacou-se, apresentando um comportamento de regime elasto-plástico acentuado. Observa-se um aumento percentual da resistência mecânica significativo de $31,5 \%$ durante o regime plástico, além do aumento da tenacidade. Este resultado confirma o comportamento mecânico peculiar apresentado pelo compósito $\mathrm{C} 3$, revelando uma forte interação entre as fases presentes.

\section{Espectroscopia na região do infravermelho (FTIR)}

A interação entre a fase polimérica e cimentícia pode ser causada pela reação de hidratação do cimento na presença da resina, com conseqüente formação de silicatos de cálcio hidratados e hidróxido de cálcio. Essa reação pode ser constatada por FTIR. Como se pode ver através da Fig. 7 para o compósito C5 (pasta de cimento pura), a presença de $\mathrm{CH}$ e C-S-H é evidente, respectivamente, a $3640 \mathrm{~cm}^{-1} \mathrm{e}$ $3460 \mathrm{~cm}^{-1}$.

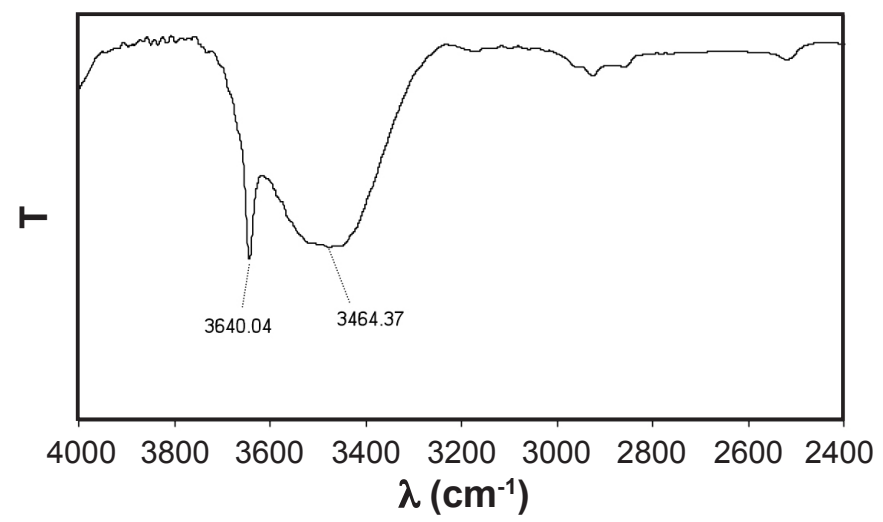

Figura 7: Espectro FTIR da pasta de cimento pura, compósito C5. [Figure 7: FTIR spectrum of the pure cemet paste, specimen C5.]

Caso haja hidratação do cimento nos compósitos C2, C3 e C4, estas duas bandas também poderão ser observadas no espectro de FTIR. Na mesma região estudada $\left(4000 \mathrm{~cm}^{-1}\right.$ a $2400 \mathrm{~cm}^{-1}$ ), a resina epoxídica (C1) apresenta distintos picos, como mostrado na Fig. 8. A banda centrada em 3443-3114 $\mathrm{cm}^{-1}$ corresponde ao estiramento da ligação O-H presente na resina epóxi, que tem região de absorção distinta do $\mathrm{O}-\mathrm{H}$ para portlandita no cimento. As demais bandas de absorção em $2958 \mathrm{~cm}^{-1}, 2928 \mathrm{~cm}^{-1}$ e $2868 \mathrm{~cm}^{-1}$ são característicos do estiramento da ligação $\mathrm{C}-\mathrm{H}$ alifático e também de um composto epoxídico [11].

Como existe uma superposição parcial da banda referente à água constituinte dos silicatos e sulphoaluminatos do cimento $\left(3460 \mathrm{~cm}^{-1}\right)$ com a banda de estiramento da ligação O-H da resina (centrado em 3414-3443 $\mathrm{cm}^{-1}$ ), o pico referente à portlandita $\left(3640 \mathrm{~cm}^{-1}\right)$ é único que pode comprovar a hidratação do cimento na presença da resina.

A Fig. 9 mostra que houve hidratação nos compósitos $\mathrm{C} 3$ e C4. O pico referente à portlandita não está claro nos compósito $\mathrm{C} 2$, que contém apenas $25 \%$ de cimento, mas é

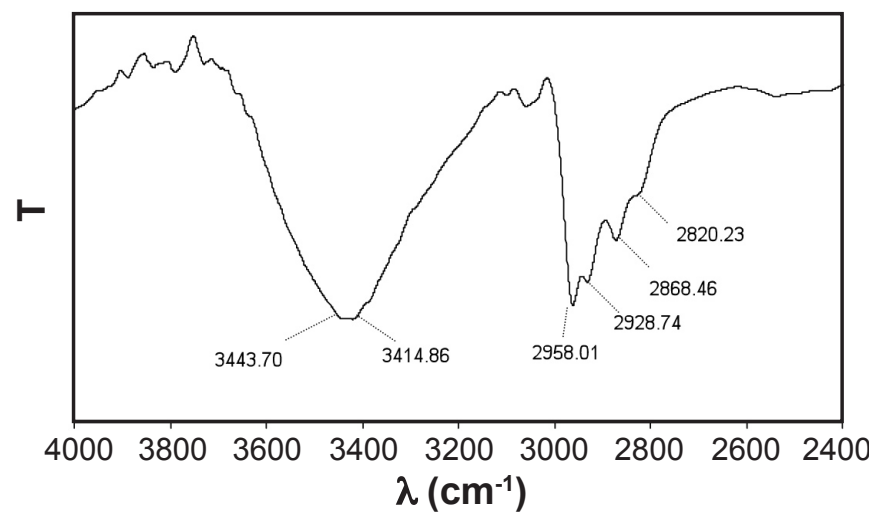

Figura 8: Espectro FTIR da resina pura, compósito $\mathrm{C} 1$.

[Figure 8: FTIR spectrum of the pure resin, specimen C1.]

bem possível que a reação também tenha ocorrido, mas a quantidade de portlandita é pequena para ser detectada por FTIR. Ainda para os compósitos C3 e C4, é possível verificar a presença de um novo pico centrado em $3410 \mathrm{~cm}^{-1}$ distinto dos demais da pasta de cimento e resina puros. A interação entre pastas de cimento e recobrimentos epoxídicos por meio de FTIR foi estudada e concluiu-se que o aparecimento de um pico próximo a $3400 \mathrm{~cm}^{-1}$ corresponde à interação (pontes de hidrogênio) entre o endurecedor da resina e à portlandita presente na pasta de cimento [12]. É possível que os picos centrados em $3410 \mathrm{~cm}^{-1}$ para os compósitos C3 e C4 sejam também resultados desta interação. De qualquer modo, a espectroscopia na região do infravermelho (FTIR) mostrou que, mesmo na ausência de água, há hidratação dos grãos de cimento para os compósitos estudados. O comportamento

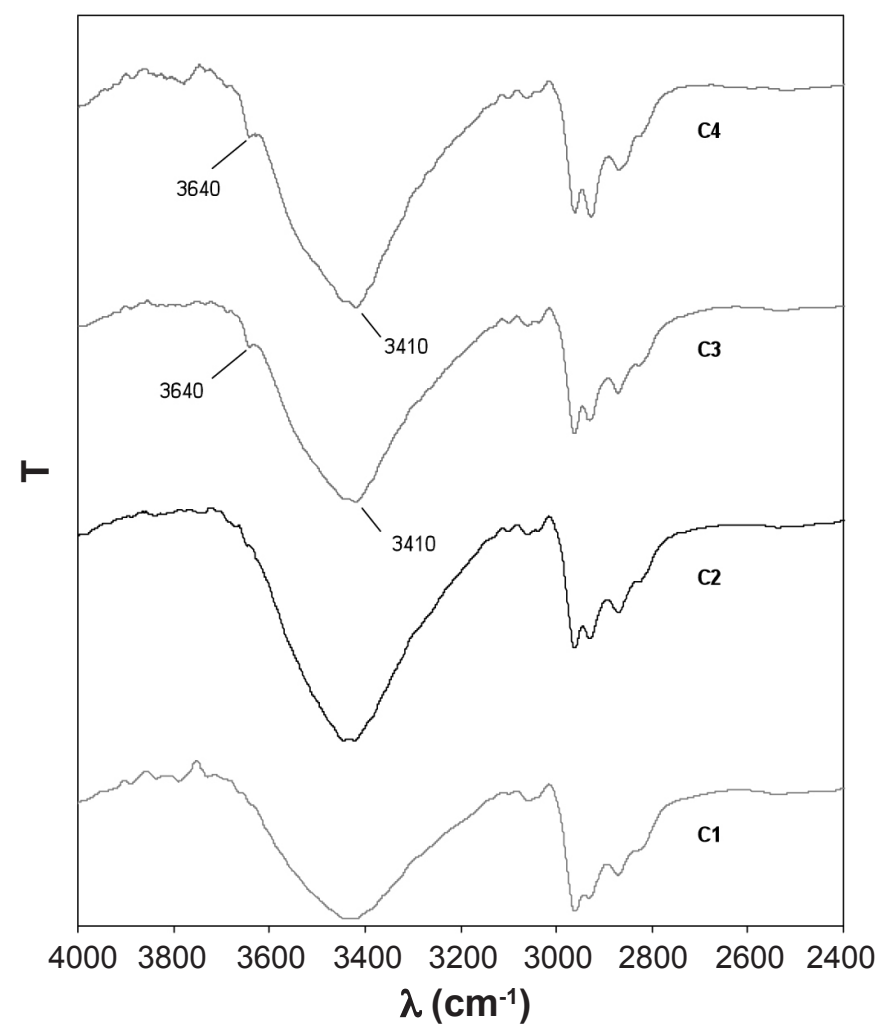

Figura 9: Espectros FTIR dos compósitos C1, C2, C3 e C4. [Figure 9: FTIR spectra of the C1,C2, C3 and C4 composites.] 
elástico distinto do compósito $\mathrm{C} 3$ não pode ser comprovado por FTIR, uma vez que os compósitos C3 e C4 possuem espectros bastante semelhantes e comportamento elásticos distintos. É provável que o compósito C3 possua uma quantidade ótima de cimento (50\%) tal que haja suficiente interação entre a resina e a pasta cimentícia formada. Por outro lado espera-se que $\mathrm{C} 3$ tenha menor porosidade que o compósito $\mathrm{C} 4$. Com isso, há menos caminhos livres para a formação de fissuras e maior elasticidade.

\section{CONCLUSÕES}

O trabalho realizado permitiu identificar o comportamento mecânico do compósito constituído de cimento Portland branco e uma fase polimérica termorrígida. A fase cimentícia promoveu um aumento da massa específica e diminuição da resistência à compressão dos compósitos. A fase cimentícia promoveu um aumento do módulo de elasticidade dos compósitos. A fase polimérica promoveu o aumento da resistência à compressão dos compósitos. O módulo de elasticidade dos compósitos variou em função da massa específica dos compósitos. O compósito C3, constituído de $50 \%$ das fases polimérica e cimentícia, exibiu a maior resistência à compressão, como também um comportamento mecânico diferenciado, exibindo elevada tenacidade. Foi observado um aumento percentual de 31,5\% da resistência mecânica durante o regime plástico. Análise por FTIR comprovou que há hidratação dos grãos de cimentos pela resina epoxídica mesmo sem adição de água. O comportamento elasto-plástico do compósito $\mathrm{C} 3$ pode ser resultante de uma interação (pontes de hidrogênio) entre a resina e portlandita formada, aliada a uma baixa porosidade que evita o início de fissuras e prolonga a elasticidade.

\section{REFERÊNCIAS}

[1] Y. Ohama, Recent Progress in Concrete-Polymer Composites, Adv. Cem. Bas. Mater. 1, 40 (1997) 5.

[2] D. Van Gemert, L. Czarnecki, M. Maultzsch, H. Schorn, A. Beeldens, P. Łukowski, E. Knapen, Cem. Concrete Comp. 27 (2005) 926-933.

[3] C. Y. Rha, C. E. Kim, C. S. Lee, K. I. Kim, S. K. Lee, Cem. Concrete Res. 29 (1999) 231-236.

[4] C. Y. Rha, J. W. Seong, C. E. Kim, S. K. Lee, W. K. Kim, J. Mater. Sci. 34 (1999) 4653-4659

[5] D. A. Silva, P. J. M. Monteiro, Cem. Concrete Res. 36 (2006) 1501-1507.

[6] A. Franceschini, S. Abramson, V. Mancini, B. Bresson, C. Chassenieux, N. Lequeux, J. Mater. Chem. 17 (2007) 913-922.

[7] U. S. Rai, R. K. Singh, Mater. Lett. 58 (2003) 235-240.

[8] E. Sakai, J. Sugita, Cem. Concrete Res. 25 (1995) 127 135.

[9] Associação Brasileira de Normas Técnicas - NRB 12989: Cimento Portland branco (1993).

[10] British Standard BS EN 12390-3, Testing hardened concrete - Compressive strength of test specimens (2002).

[11] Federation of Societies for Coating Technology, An Infrared Spectroscopy Atlas for the coating industry, $4^{\text {th }} \mathrm{Ed}$., 1 (1991).

[12] F. Djouani, C. Connan, M. M. Chehimi, K. Benzarti, Surf. Interface Anal. 40 (2008) 146-150.

(Rec.07/11/2008, Rev. 29/04/2009, Ac. 08/09/2009) 